\title{
Anatomical Variations of the Cystic Duct in Turkish Population and their Association with Biliary Track Stone
}

\author{
Yaşar Taştemur \\ Department of Anatomy, Sivas Cumhuriyet University Medical Faculty, Sivas, Turkey
}

\begin{abstract}
Objective: To evaluate the anatomy and various anatomical variations of the cystic duct and their association with the stones in the biliary tract in the Turkish population.

Study Design: Observational study.

Place and Duration of Study: Sivas Cumhuriyet University Hospital between November 2017 and August 2019.

Methodology: Patients who had undergone MRCP procedures at the study centre were assessed retrospectively. MRCP images were used to evaluate the variations of the cystic duct. Association with the stones in the biliary tract was noted with $p<0.05$ as significant.

Results: Three thousand MRCPs were evaluated. Among the 930 patients included in the study, 408 were males (43.9\%), 61.9 \pm 17 years, while 522 were females $(56.1 \%), 57.1 \pm 19.2$ years. The most common variation was lateral insertion in 372 patients $(40 \%)$, medial insertion in 226 patients $(24.3 \%)$, and high insertion in 137 patients (14.7\%). Lateral, medial, high insertions (all $p<0.001)$, parallel course of the cystic duct $(p<0.001)$, low medial $(p=0.024)$, and posterior insertion $(p=0.003)$ were significantly associated with the calculi in the biliary tract. The highest coexistence frequency was at anterior, posterior, and low medial insertion variation groups, at $25 \%, 23.8 \%$, and $25 \%$, respectively.

Conclusion: Preoperative information of anatomical variations of the cystic duct is not only important for operative planning; but some variations are significantly associated with the cholelithiasis and/or choledocholithiasis.
\end{abstract}

Key Words: Cystic duct, Variations, Bile stones, Cholelithiasis, Choledocholithiasis.

How to cite this article: Taştemur Y. Anatomical Variations of the Cystic Duct in Turkish Population and their Association with Biliary Track Stone. J Coll Physicians Surg Pak 2020; 30(10):1005-1008 .

\section{INTRODUCTION}

Although the anatomy and variations of the biliary system are frequently studied and well established, ${ }^{1,2}$ it is still challenging to understand and predict, due to the very close proximity of the major organs and complex embryogenesis processes. Subsequently, for both surgical and medical purposes, the site's anatomy and variations must be known in detail. ${ }^{3} \mathrm{An}$ important cause of bile duct injury is the failure to identify the ductal anatomy, particularly in anatomical variants. The complete transaction of the common bile duct (CBD) occurs when CBD is mistaken for the cystic duct; and it is one of the dreaded complications of laparoscopic and open cholecystectomies. ${ }^{4}$

Variations may not always be related to congenital reasons. The biliary tract is under threat of iatrogenic damage, including from open or laparoscopic surgery, which can also change the anatomy of the biliary track and create unique variations.

Correspondence to: Dr. Yaşar Taştemur, Department of Anatomy, Sivas Cumhuriyet University Medical Faculty, Sivas, Turkey

E-mail:md.tastemur.yasar@gmail.com

Received: February 20, 2020; Revised: July 27, 2020;

Accepted: August 21, 2020

DOI: https://doi.org/10.29271/jcpsp.2020.10.1005
As iatrogenic damage may end up as adhesions, thickening of the tissues, inflammation, and bleeding, it can result in the need for a cholecystectomy, liver resection surgery or liver transplantation from live donors. ${ }^{5}$

Different cystic duct variations are described in the previous studies based on its length, course, and site of insertion with a common hepatic duct (CHD). Some varieties that are clinically significant include low insertion of the cystic duct, parallel course of the cystic duct with CHD, anterior or posterior spiral course with medial insertion, absent or short cystic duct (length $<5 \mathrm{~mm}$ ), aberrant drainage of cystic duct to the right hepatic or left hepatic duct, aberrant or accessory intrahepatic ducts draining into the cystic duct, and double cystic duct. ${ }^{6,7}$

USGs, CTs, and cholescintigraphies play an essential role in the evaluation of suspected biliary anomalies. Conventional MRI sequences allow additional evaluation of the biliary system and adjacentstructures. However, MRCPs are used to evaluate inadequate findings, and provide a comprehensive and non-invasive assessment of the biliary tract and gallbladder. ${ }^{8,9}$ Indeed, MRCPs are widely used to evaluate the gall tree and used in place of a diagnostic ERCPand PTC for many patients.

The standard biliary anatomy and its variations should be considered, especially before invasive procedures and surgery. For this 
purpose, MRCP findings, which are helpful in preoperative planning and are a non-invasive method, are essential.

The primary aim of this study was to evaluate the anatomy and various anatomical variations and imaging features of the cystic duct by using MRCP. The secondary aim of the study was to find whether these variations in the cystic duct have had any effect on gall stone formation.
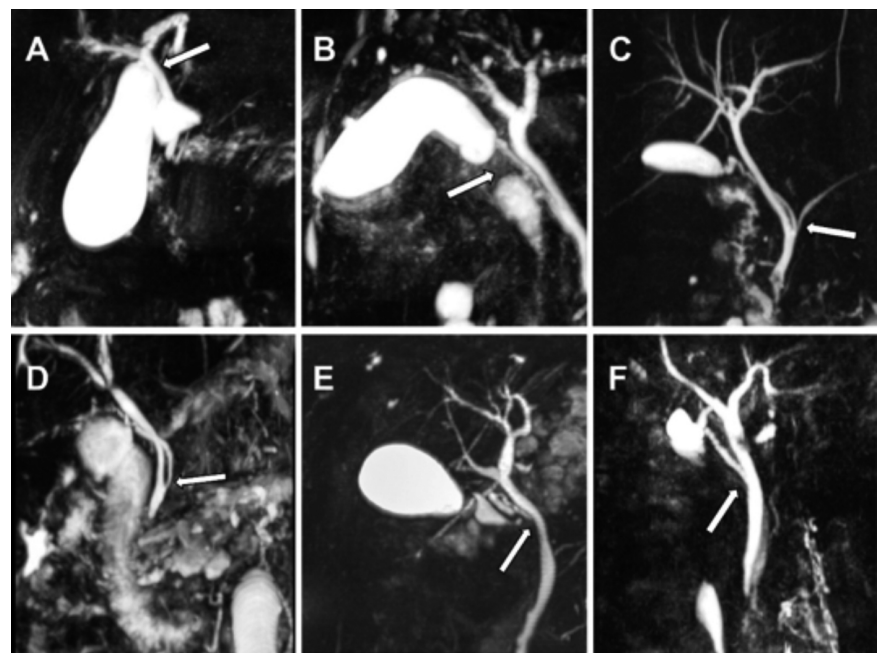

Figure 1a: MRCP images of: (A) Short, (B) Parallel, (C) Medial, (D) Low Medial, (E) High, (F) Lateral (Normal) variations of the biliary system.

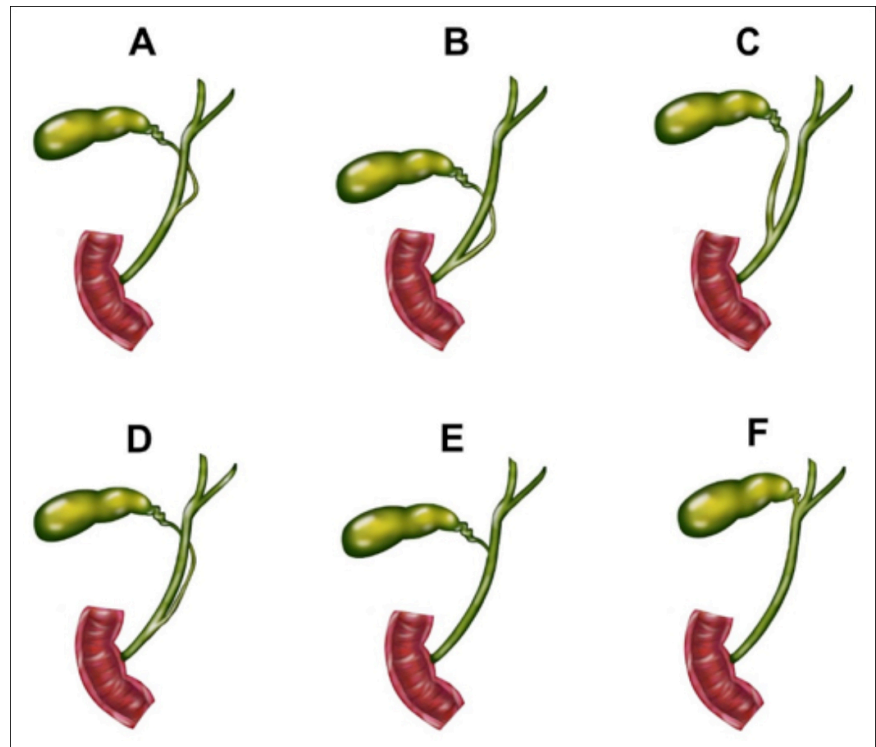

Figure 1b: Illustrations of: (A) Medial, (B) Low medial, (C) Lateral (normal), (D) Parallel, (E) High, (F) Short of the biliary system. ${ }^{16}$

\section{METHODOLOGY}

Patients who had undergone MRCP procedures at the Sivas Cumhuriyet University Hospital between November 2017 and August 2019 were assessed respectively. There was no age limit. The exclusion criteria were history of surgery on the biliary tract except for cholecystectomy, whose images could only be inadequately evaluated due to significant respiratory artefacts during the imaging, those with prosthetics incompatible with the MRI, having pacemakers or metallic stents, choledochal cysts, cholangiocarcinoma, liver transplantation, pancreas head tumors, or multiple livermass.
Ethics Committee approval was obtained from the University Ethics Committee before the start of the study. MRI examinations were performed using a 1.5 Tesla (T) unit (Siemens, Acra, Germany) with a body coil. Patients had fasted for 6 hours before the MRCP. No contrast agent was given intravenously or orally. All images were obtained in the supine position.

Data was expressed as mean \pm SD and values (min-max). The frequency variations described in the present study are expressed as percentages. The Chi-square test was applied on categorical variables. Any value less than $0.05(p<0.05)$ was considered significant. The collected data were analysed using SPSS software (Version 22).

\section{RESULTS}

Among the 930 patients included in the study, 408 were males $(43.9 \%)$, while 522 were females $(56.1 \%)$. The mean age was $61.9 \pm 17$ years in males and $57.1 \pm 19.2$ years in females, ranging from 23 to 92 years. The most common variation was lateral insertion, which was observed in 372 patients (40\%).

Lateral insertion was followed by medial insertion observed in 226 patients (24.3\%). The third most common variation was the high insertion in 137 patients (14.7\%). The variations were diverse and multiple (Figure $1 \mathrm{a}$ and $1 \mathrm{~b}$ ).

Nevertheless, the rest of the variations were by far rarer than those of the above three. The frequencies of all variations are summarised in Table I. Some cystic duct variations, such as the lateral insertion and medial insertion, were not only the frequent ones; but also the ones significantly associated with the highest gallbladder stone frequencies in their variations with $40.3 \%$ and $42.5 \%$, respectively (Table I). Lateral, medial, high insertions (all $p<0.001)$, parallel course of the cystic duct $(p<0.001)$, low medial $(p=0.024)$, and posterior insertion $(p=0.003)$ were significantly associated with the calculi in the biliary tract.

The coexistence of choledochal and gallbladder stones was also showed in this study. Interestingly, the highest coexistence frequency was at anterior, posterior, and low medial insertion variation groups, $25 \%, 23.8 \%$, and $25 \%$, respectively (Tablell).

Table I: Frequencies and percentages of biliary system variations.

\begin{tabular}{|l|c|c|}
\hline & Frequency & $\begin{array}{c}\text { Percentages } \\
\text { (\%) }\end{array}$ \\
\hline Lateral insertion & 372 & 40 \\
\hline Medial insertion & 226 & 24.3 \\
\hline Low medial insertion & 44 & 4.7 \\
\hline The parallel course of thecystic duct & 50 & 5.4 \\
\hline High insertion & 137 & 14.7 \\
\hline Short cystic duct & 5 & .5 \\
\hline Aberrant insertion & 13 & 1.4 \\
\hline Other variations & 12 & 1.3 \\
\hline Posterior insertion & 63 & 6.8 \\
\hline Anterior insertion & 8 & .9 \\
\hline Total & 930 & 100.0 \\
\hline
\end{tabular}


Table II: Choledochal stones and gallbladder stones with the variations of the biliary system.

\begin{tabular}{|c|c|c|c|c|c|c|c|c|c|}
\hline & No Stone & $\%$ & Choledochal stone & $\%$ & Gallbladder stone & $\%$ & $\begin{array}{l}\mathrm{C}+\mathrm{G} \\
\text { stone }\end{array}$ & $\%$ & $\mathbf{p}$ \\
\hline Lateral insertion & 160 & 43.0 & 11 & 3.0 & 150 & 40.3 & 51 & 13.7 & $<0.001$ \\
\hline Medial insertion & 89 & 39.4 & 5 & 2.2 & 96 & 42.5 & 36 & 15.9 & $<0.001$ \\
\hline Low medial insertion & 17 & 38.6 & 3 & 6.8 & 13 & 29.5 & 11 & 25 & 0.024 \\
\hline The parallel course of the cystic duct & 20 & 40 & 1 & 2 & 20 & 40 & 9 & 18 & $<0.001$ \\
\hline High insertion & 63 & 46 & 4 & 2.9 & 47 & 34.3 & 23 & 16.8 & $<0.001$ \\
\hline Short cystic duct & 4 & 80 & 0 & 0 & 1 & 20 & 0 & 0 & 0.180 \\
\hline Aberrant insertion & 8 & 61.5 & 0 & 0 & 4 & 30.8 & 1 & 7.7 & 0.058 \\
\hline Other variations & 6 & 54.5 & 0 & 0 & 5 & 45.5 & 0 & 0 & 0.763 \\
\hline Posterior insertion & 23 & 36.5 & 4 & 6.3 & 21 & 33.3 & 15 & 23.8 & 0.003 \\
\hline Anterior insertion & 3 & 37.5 & 0 & 0 & 3 & 37.5 & 2 & 25 & 0.882 \\
\hline
\end{tabular}

\section{DISCUSSION}

Since the present study focusses on the variations of the cystic duct, it is worth mentioning that the sample size is one of the most crucial factors in variation studies. ${ }^{10-12}$ Sarawagi et al. conducted a similar study among 198 patients in 2016. ${ }^{13}$ Lateral insertion (51.5\%) accounted for the highest variation in their study, which was referred to as normal by the authors. Similarly, lateral insertion also had the most frequent variation in the present study (40\%).

Nonetheless, posterior insertion was markedly higher $(6.8 \%$ vs. $20.2 \%)$. The rest of the variations were quite similar in terms of percentages. The main difference between the studies are the patient numbers (930 vs. 198) in the studies, and perhaps the difference in nationalit, since the other study was conducted in India.

In another study conducted in Turkey with 115 patients, variations of the cystic duct were investigated. ${ }^{8}$ This study took 475 patients under study and decided that only 115 patients had a variation in the biliary system. The lateral insertion was found to be one of the dominant variations. They observed a standard deviation of $76 \%$ of patients, which is much higher than in the present study, where more diverse variations were found. On the other hand, the frequency of the many variations, including posterior insertion, aberrant insertion, and the short cystic duct was similar. The only significant difference was medial insertion (24.3\% vs. 3.8).

In another study from Turkey, Onder et al. investigated the differences in biliary tract variations in 2013. They included 590 patients and detected variations in 239 patients (39\%). Since they also did not mention what the norm was, they found that $61 \%$ of patients were standard, which is much higher than in the present our study (61\% vs. 40\%). When the variation frequency of the study is compared to the present study, the frequency distal medial insertion and short cystic were comparable ( $6.8 \%$ vs. $4.8 \%),(1.7 \%$ vs. $0.5 \%)$, respectively. On the other hand, medial insertion was significantly more frequent in the present study $(9.8 \%$ vs. $24.3 \%) .3$

Mortele et al. published a review about the anatomic variants of the biliary tree in $2001 .{ }^{11}$ They mentioned the most observed frequencies of all variations presented in the present study, finding $58 \%$ for normal biliary anatomy, which is close to this research results. The rate of the remaining variations was almost the same as in this study.

The data of this study may have clinical significance in many ways. The three biliary system variations have clinical importance, which include the medial insertion, low insertion, and parallel course of the cystic duct. Possibly one of the most important findings of this study was the high rates of medial insertion. One of the major causes of bile duct injury is the failure to identify the ductal anatomy, particularly in the presence of anatomical variants. A complete transaction of the common bile duct occurs when CBD is mistaken for a cystic duct. It is one of the most dreaded complications of laparoscopic and open cholecystectomy. ${ }^{4}$ It is known regarding medial insertion that a cystic duct should be left long remnant during cholecystectomy operations because the dissection of the medial insertion cystic duct up to the end, may cause injuries. Low insertion of the cystic duct is associated with a high rate of CBD stone formation and a higher recurrence of $C B D$ stones. Failure to identify a low insertion of the cystic duct may result in technical difficulties during ERCP procedures which may lead to complications. ${ }^{14}$

In the present study, the associations of choledochal and gallbladder stone frequencies were also investigated together with anatomical variations. As far as the authors of the present study know, this is the first study in the literature investigating the relationship of stone frequencies with anatomical variations. The clinical perspective must estimate the potential presence of biliary system stones for proper clinical decision-making. Both lateral and medial insertion variances were the most common; and tended to produce stones in the gallbladder. Furthermore, the cystic duct's parallel course also had very high gallbladder stone frequencies but with a gradually low total frequency. When those three variations are detected on preoperative imaging, the clinician should also look for the stone in the biliary system.

There is a minimal amount of information about the relationship of anatomical variations of the cystic duct and choledochal stones. Tsiridis et al. and Kao et al. suggested that patients with low insertion variations tend to have a higher 
incidence of choledochal stones. ${ }^{14,15}$ Those studies are in accordance with the present study's results. It was found in the present study that lower insertion and posterior insertions tend to produce choledochal stones when compared to other variations. This finding may have a clinical significance since lower insertion variation creates difficulty in ERCP operations and may result in a higher incidence of complications. ${ }^{16}$

In the current study, the coexistence of choledochal and gallbladder stones were also investigated. The highest coexistence frequency was among the anterior, posterior, and low medial insertion variation groups, which means when the clinician detected a stone in one part of the biliary system, he/she should also check for other possible stone locations.

It is evident that knowing the anatomy of the biliary system and being able to identify the variations would greatly benefit surgeons, leading to a reduction of complications. However, it is hard to classify the variations and conduct comparative studies among a large number of patients. The present study is conducted on a very large patient population, and it is believed it illuminates the main variations of the anatomical site.

\section{CONCLUSION}

Accurately knowing anatomy of the cystic duct is essential for surgery that is more precise. There is an association between the cystic duct variations and the occurrence of the gall bladder and bile duct stones.

\section{ETHICAL APPROVAL:}

The present study is approved by the Sivas Cumhuriyet University Clinical Research Ethical Committee.

\section{PATIENTS' CONSENT:}

Since the study is conducted retrospectively, getting the patient consent in person was not applicable. Nonetheless, even before the Ethical Committee application, proper authorisations were taken from Sivas Cumhuriyet University Hospital administration by ensuring the patients' anonymity and protection of the patient data.

\section{CONFLICT OF INTEREST:}

There is no conflict of interest to declare.

\section{AUTHOR'S CONTRIBUTION:}

YT: Made all evaluations and analysed the data.

\section{REFERENCES}

1. Muraki T, Reid MD, Pehlivanoglu B, Gonzalez RS, Sekhar A, Memis $B$, et al. Variant anatomy of the biliary system as a cause of pancreatic and peri-ampullary cancers. HPB (Oxford) 2020; S1365-182X(20)30096-4. doi: 10.1016/j.hpb.2020. 03.014.

2. Boukoucha M, Dhieb F, Khelifa RB, Znaidi H, Elaifi R, Daghfous $A$. Cholecystitis on gallbladder duplication: A case report and literature review. Int J Surg Case Rep 2020; 72:406-10. DOI: 10.1016/j.ijscr.2020.06.053.

3. Önder H, Özdemir MS, Tekbas G, Ekici F, Gümüs H, Bilici A. 3T MRI of the biliary tract variations. Surg Radiol Anat 2013; 35(2):161-7. DOI: 10.1007/s00276-012-1021-0.

4. Canullán C, Baglietto N, Merchán Del Hierro P, Petracchi E. Ten strategies to improve the efficacy of laparoscopic biliary surgery. Cir Esp 2020; 16:S0009-739X(20)30198-6. DOI: 10.1016/j.ciresp.2020.05.027.

5. Heller SL, Lee VS. MR imaging of the gallbladder and biliary system. Mag Reson Imaging Clin N Am 2005; 13(2): 295-311. DOI: 10.1016/j.mric.2005.03.003.

6. Wu YH, Liu ZS, Mrikhi R, Ai ZL, Sun Q, Bangoura G, et al. Anatomical variations of the cystic duct: Two case reports. World J Gastroenterol 2008; 14(1):155-7. DOI: 10.3748/ wjg.14.155.

7. Hashimoto $M$, Hashimoto $M$, Ishikawa $T$, lizuka $T$, Matsuda $M$, Watanabe G. Right hepatic duct emptying into the cystic duct. Surgical Endoscopy 2002; 16(2):359. DOI: 10.1007/ s004640041029.

8. Düşünceli E, Erden A, Erden I. Anatomic variations of the bile ducts: MRCP findings | Biliyer sistemin anatomik varyasyonlari: MRKP bulgulari. Tani Girisim Radyol 2004; 10(4): 296-303.

9. Yam BL, Siegelman ES. MR imaging of the biliary system. Radiol Clin North Am 2014; 52(4):725-55.

10. Dohke M, Watanabe Y, Okumura A, Amoh Y, Oda K, Ishimori $\mathrm{T}$, et al. Anomalies and anatomic variants of the biliary tree revealed by MR cholangiopancreatography. Am J Roentgenol 1999; 173(5):1251-4. DOI: 10.2214/ajr.173.5. 10541098.

11. Mortelé KJ, Ros PR. Pictorial essay. Anatomic variants of the biliary tree: MR cholangiographic findings and clinical applications. Am J Roentgenol 2001; 177(2):389-94. DOI: 10.2214/ajr.177.2.1770389.

12. Turner MA, Fulcher AS. The cystic duct: Normal anatomy and disease processes. Radiographics 2001; 21(1):3-22. DOI: 10.1148/radiographics.21.1.g01ja093.

13. Sarawagi R, Sundar S, Gupta SK, Raghuwanshi S. Anatomical variations of cystic ducts in magnetic resonance cholangiopancreatography and clinical implications. Radiol Res Pract 2016; 2016: 3021484. DOI: 10.1155/2016/3021484.

14. Tsitouridis I, Lazaraki G, Papastergiou C, Pagalos E, Germanidis G. Low conjunction of the cystic duct with the common bile duct: Does it correlate with the formation of common bile duct stones? Surg Endosc Other Interv Tech 2007; 21(1):48-52. DOI: 10.1007/s00464-005-0498-6.

15. Kao JT, Kuo CM, Chiu YC, Changchien CS, Kuo CH. Congenital Anomaly of Low Insertion of Cystic Duct Endoscopic Retrograde Cholangiopancreatography Findings and clinical significance. J Clin Gastroenterol 2011; 45(7): 626-9. DOI: 10.1097/ MCG.0b013e31821bf824.

16. George RA, Debnath J, Singh K, Satija L, Bhargava S, Vaidya A. Low insertion of a cystic duct into the common bile duct as a cause for a malpositioned biliary stent: Demonstration with multidetector computed tomography. Singapore Med J 2009; 50(7):243-6. 